\title{
Desigualdad de género en la entrega de los Premios Nobel 1901-2020
}

\author{
Gender inequality in the Nobel Prizes winners 1901-2020 \\ Dulce María del Carmen Delgadillo Álvarez ${ }^{1}$
}

Fecha de recepción: 27-05-2020

Fecha de aceptación: 16-3-21

\begin{abstract}
Resumen
La participación femenina en la investigación científica ha sido poco valorada. Esto lo demuestra la entrega del Premio Nobel, máximo galardón otorgado para laurear el trabajo de eminentes personajes del mundo académico. Existe una evidente desigualdad de género en el otorgamiento anual de esta distinción pues, de 1901 a 2020, solamente 23 mujeres la han obtenido en las áreas científicas y solo tres de ellas la han ganado en solitario, puesto que el resto la han compartido. Esto se debe, en parte, a las pocas oportunidades que tenían las mujeres en el siglo pasado para acceder a la educación superior y para realizar investigaciones científicas y, a pesar de que esto ha cambiado con el transcurrir de los años hasta los albores del nuevo milenio, el número de mujeres científicas aún es bajo. El objetivo de este trabajo es realizar una revisión de los logros de algunas mujeres científicas a lo largo de la historia y evidenciar el poco reconocimiento que se les ha dado. Concretamente en el caso del reconocimiento a mujeres a partir del siglo XX, se extrajeron los datos registrados en el sitio web del Premio Nobel (Nobel Prize, 2021) y se realizó un análisis para determinar la frecuencia con la que el galardón ha sido entregado a mujeres en las áreas de Física, Química, Fisiología y Medicina. Se realizó también una breve revisión bibliográfica sobre la obra de las ganadoras, así como de algunas eminentes mujeres que dedicaron su vida a la ciencia y que tuvieron importantes logros en ella, aunque no fueron reconocidas en su momento.
\end{abstract}

Palabras clave: Mujer, investigación, científicas, Premio Nobel, desigualdad.

\begin{abstract}
Female participation in scientific research has been undervalued. This is demonstrated on the Nobel Prize, the highest award given to honor the work of eminent figures from the academic world. There is an evident gender inequality in the annual granting of this distinction because, from 1901 to 2020, only 23 women have obtained it in scientific areas and only three of them have won it alone since the rest have shared it. This is partially due to the few opportunities' women had in the last century to access higher education and to conduct scientific research and, although this has changed over the years until the dawn of the new millennium, the number of female scientists is still low. The objective of this work is to perform a review of the achievements of some women scientists throughout history and to show the little recognition that they have been given. Specifically, in the case of the recognition of women scientists from the 2oth century, the data registered on the Nobel Prize website (The Nobel Prize, 2021) were extracted and an analysis was carried out to determine the frequency with which the award has been given to women in the areas of physics, chemistry, and physiology or medicine. A brief bibliographic review was also accomplished on the work of the winners, as well as some eminent women who dedicated their lives to science and who had important achievements in it, although they were not recognized at their time.
\end{abstract}

Keywords: Woman, research, scientist, Nobel Prize, inequality.

1 Doctora en Ciencias en Biomedicina Molecular por el Instituto Politécnico Nacional (IPN-México). Laboratorio Nacional de Servicios Experimentales del Centro de Investigación y de Estudios Avanzados-IPN (LaNSE-Cinvestav), México. Correo electrónico: cdelgadillo@cinvestav. mx. Orcid: 0000-0002-5057-7727 


\section{Introducción}

La investigación científica -referida específicamente a las ciencias exactas y naturales- es la disciplina encargada de buscar la explicación de fenómenos naturales mediante la aplicación del método científico. Sus resultados son descubrimientos que propician avances que ayudan a entender el universo y están encaminados a la resolución de problemas que afectan a la humanidad y a la naturaleza. Durante siglos, las personas que han realizado la investigación científica han sido mayoritariamente hombres, pero también ha habido, y hay, eminentes mujeres dedicadas a esta disciplina, aunque su participación no siempre ha contado con el reconocimiento debido.

En casi todo el mundo y en casi todas las épocas, se ha creído que las mujeres son incapaces de incursionar en la ciencia del mismo modo que los hombres. Un ejemplo reciente de esto ocurrió en el año 2005, cuando el entonces presidente de la Universidad de Harvard, Lawrence Summers, manifestó en una conferencia que si las mujeres no lograban llegar a lo más alto en matemáticas, ciencias e ingeniería se debía a una incapacidad innata en ellas. Summers sustentó su aseveración diciendo que, entre otras cosas, las mujeres no quieren trabajar 80 horas a la semana y que tienen pequeñas diferencias de aptitud o capacidad media en matemáticas o en el área científica que se traducen en una gran disparidad en el nivel intelectual que se necesita para hacer ciencia (García Dauder y Pérez Sedeño, 2017).

Cuando el ser humano dejó de ser nómada, el sedentarismo promovió el establecimiento de sociedades, donde muchas de ellas implantaron una dominación física, sexual, social y, por supuesto, intelectual, que certificaba el control de la mujer por parte de los hombres y que ha permanecido por siglos (Duarte Cruz y García-Horta, 2016). Aunado a esto, la desigualdad en las oportunidades laborales hacia las mujeres y el llamado "techo de cristal", definitiva como la barrera invisible que impide que mujeres altamente cualificadas y con un nivel de competencia elevado avancen en sus carreras, son resultado de una cultura y una sociedad patriarcal y androcéntrica imperante prácticamente en todo el mundo (Camarena Adame y Saavedra García, 2018).
Si bien con el correr de los años las barreras para acceder a la educación se han reducido, muchas mujeres han sido cubiertas por la sombra de hombres cercanos a ellas. Otras, aunque muy pocas, afortunadamente han sido galardonadas por su labor académica. El objetivo de este trabajo es hacer una revisión de los logros de algunas mujeres científicas a lo largo de la historia y hacer notar el poco reconocimiento que han tenido a su trabajo.

Con la revisión de datos registrados en la página del Premio Nobel (Nobel Prize, 2021), se realizó un análisis para determinar la frecuencia con la que el galardón ha sido entregado a mujeres, desde su instauración hasta el año 2020, en las áreas de Física, Química y Fisiología o Medicina. La obra de estas y otras mujeres científicas que dedicaron su vida a la ciencia dan testimonio de sus capacidades intelectuales y académicas y son un ejemplo no solo para las nuevas generaciones de niñas y adolescentes sino también para todos los seres humanos.

\section{Algunas mujeres científicas en la historia}

La ciencia se ha desarrollado con la participación femenina a pesar del limitado acceso a la educación que han tenido las mujeres por ser consideradas seres con poca capacidad de aprendizaje y aptas solo para fines reproductivos (García Dauder y Pérez Sedeño, 2017). No obstante, la ciencia ha contado con mujeres inteligentes y audaces, algunas de ellas se mencionan a continuación.

Hipatia fue una famosa filosofa líder de los neoplatónicos alejandrinos en el año 40o. Siendo no cristiana, sus clases y conferencias eran escuchadas también por cristianos. Sus conocimientos matemáticos y astronómicos la llevaron a mejorar el astrolabio e inventar un densímetro. Hipatia era un modelo de valor ético, rectitud, veracidad, dedicación cívica y proezas intelectuales que despertó la envidia de Cirilo, patriarca de Alejandría, quien además tenía conflictos con el prefecto de la ciudad Orestes, exalumno y amigo de Hipatia. Dada la cercanía con Orestes, Cirilo señaló a Hipatia como el obstáculo en su relación con el prefecto e hizo circular el rumor de que la maestra realizaba actividades satánicas y de brujería. En un día de marzo del año 414, una multitud dirigida por Pedro, lector de la iglesia, sacó a Hipatia de su carruaje 
y la arrastró hasta la iglesia del Cesarión donde fue desmembrada. Luego, su cuerpo fue quemado en una pira fuera de la ciudad, en un lugar llamado Cinaron. El asesinato de Hipatia provocó que la ciencia, la filosofía y la vida intelectual de Alejandría se apagaran, y desde ese trágico día, Europa prácticamente se sumió en la oscuridad, hasta la llegada de la Ilustración (Dzielska, 2004).

No obstante, el oscurantismo del Medievo tuvo algunas excepciones. El Sacro Imperio Romano Germánico contó con Hildegarda de Bingen (1098-1179). dedicada al servicio de Dios, Hildegarda escribió textos religiosos y obras musicales y científicas, entre estas últimas está Liber subtilitatum diversarum naturarum creaturarum (Libro sobre las propiedades naturales de las cosas creadas) que en el siglo XIII fue dividido en dos textos: Physica (Historia Natural), y Causae et Curae (Problemas y Remedios) que también son conocidos como Libro de la Medicina Sencilla y Libro de la Medicina Compleja (de Bingen).

Otra excepción al oscurantismo se dio en Italia ya que, en 1338 , las autoridades consideraron que elevar el nivel de educación de las mujeres favorecía a sus familias y a su comunidad pues las preparaba mejor para ser esposas y madres. Así, llamaron mujeres excepcionales a aquellas que rompían con el estereotipo de inferioridad que la sociedad de ese tiempo tenía de las féminas. Las mujeres excepcionales usualmente pertenecían a familias poderosas, a la aristocracia urbana o a la élite profesional y, si bien recibían instrucción en latín y/o griego, historia, filosofía y literatura, pocas estudiaron matemáticas y ciencias. Pero era mucho más de lo que se podía ver en otros lugares de Europa. Un par de ejemplos de mujeres excepcionales son: Laura Ceretta (1469-1499) y Cassandra Fedele (1465-1558). La primera estudió matemáticas y astronomía e inició debates a través de cartas a intelectuales de su época, en las que cuestionó el papel tradicional de las mujeres y la opresión para restringirlas sólo al matrimonio y la maternidad. Cassandra participó en debates en el Senado Veneciano en el que defendió la educación de las mujeres (Frize, 2013).

A pesar de que en el resto de Europa las mujeres no tenían oportunidad de acceder a una educación formal, algunas niñas tuvieron la suerte de contar con padres que apoyaron su desarrollo intelectual. Por ejemplo, Marie Cunitz (1610-1664), fue una astrónoma que nació en Polonia. Marie publicó el libro Urania propitia, en el que tradujo de manera simple las leyes de Kepler y corrigió errores matemáticos en estas (Frize, 2013). En Alemania, Maria Eimmart (1676-1707) y Maria Winkelmann (16701720), también se dedicaron a la astronomía. Eimmart como ayudante de su padre primero y luego de su esposo, ambos astrónomos, creó numerosas ilustraciones astronómicas exactas y realizó más de 350 dibujos de las fases de la luna que sirvieron para hacer un mapa lunar e ilustró además el eclipse total de 1706. Winkelmann también fue asistente de un astrónomo antes de casarse con otro a quién ayudó en su observatorio de Berlín, donde descubrió un cometa. En los dos casos, sus trabajos fueron publicados bajo el nombre de los hombres con los que laboraron (Frize, 2013).

En Francia, Gabrielle-Emilie le Tonelier de Bretuil (17061749), mejor conocida con su nombre de casada: marquesa Du Châtelet, tuvo la misma educación que sus hermanos y su pertenencia a la aristocracia le permitió, aún después del matrimonio y del nacimiento de sus hijos, continuar con sus estudios e interactuar con hombres letrados de la época. Madame Du Châtelet tradujo los Philosophiae naturalis principia mathematica de Newton e hizo una versión comentada en francés. Trabajó en la traducción de La fábula de las abejas de Bernard Mandeville, y escribió un Prefacio en el que expresó su sentir sobre el papel de las mujeres de su época y defendió la necesidad de la educación femenina. En 1737 presentó el trabajo Disertación sobre la naturaleza y la propagación del fuego en un concurso convocado por la Academia de Ciencias de París. En 1740, Madame Du Châtelet publicó un amplio y denso tratado sobre el estado de la física en la época: Les Institutions de Phisique. Esta obra le dio a la marquesa reconocimiento en el mundo de la ciencia y le permitió tener debates científicos con miembros de la Academia (du Châtelet, 1996).

En Inglaterra, Augusta Ada Byron, conocida como Ada Lovelace (1815-1852), fue la única hija legítima del poeta lord Byron. La madre de Ada puso especial empeño en que aprendiese matemáticas pues trataba de reprimir 
su imaginación (que juzgaba peligrosa y dañina por venir de los Byron). Dada su posición social, Ada tuvo contacto con personajes importantes de su época como el matemático Charles Babbage, quien desarrollaba entonces una máquina analítica. Ada vislumbró en este invento el potencial de digitalizar la información y escribió un esbozo de lo que ahora conocemos como un programa informático, algo que ni Babbage ni ningún colaborador suyo imaginaba (Essinger, 2014).

En Francia, Alemania e Inglaterra mujeres como las mencionadas tuvieron acceso a la educación e hicieron contribuciones serias a la ciencia, pero ninguna de las ellas fue admitida en las Academias de Ciencias de los países en los que nacieron, vivieron y trabajaron (Frize, 2013).

En abril de 1732 Laura Maria Catherina Bassi (1711-1778) completó sus estudios en la Universidad de Bolonia y fue, luego de siete siglos de historia de la institución, la primera mujer -en toda Europa- que obtuvo un doctorado, que fue invitada a unirse como miembro a la Academia de Ciencias de Bolonia y que logró un puesto como profesora honoraria de la misma Universidad, y si bien al principio su sueldo anual fue de 500 liras, llegó a elevarse a 1200, el más alto que la Universidad pagaba. Sin embargo, a pesar de su empleo académico, Laura no podía impartir clases públicamente por lo que realizaba su labor docente en su casa, así como experimentos sobre posibles aplicaciones medicinales de la electricidad. Esto último en colaboración con su esposo, un profesor de la misma Universidad, quien fungió como su ayudante. En 1776, el Senado de Bolonia le concedió la cátedra de física experimental en el Instituto de Ciencias y con esto se convirtió en la primera mujer nombrada para una cátedra de física en una universidad europea. No obstante, a pesar de ser parte del exclusivo grupo de 25 científicos de la Academia de Ciencias de Bolonia, el nombramiento de Laura Bassi fue controversial y nunca tuvo los mismos privilegios que sus compañeros (Gregersen, 2011; Frize, 2013).

El tiempo continuó y, luego de que en las primeras décadas del siglo XX se demostrara la importancia del ácido desoxirribonucleico (ADN) como el material que almacena y transmite la información genética de todos los organismos (Griffith, 1928), la elucidación de su estructura molecular se convirtió en el objetivo de varios grupos de investigación científica en el mundo. En uno de esos grupos trabajaba como asociada de investigación la química y cristalógrafa inglesa Rosalind Elsie Franklin, en la Unidad de Biofísica del Consejo de Investigación Médica del King's College en Londres, bajo la dirección de John Randall. En el mismo lugar trabajaban Maurice Wilkins y Raymond Gosling; ambos habían iniciado un análisis de ADN por difracción de rayos X casi un año antes de la incorporación de Rosalind a la Unidad. Dada la experiencia de Rosalind con experimentos de difracción de rayos X, Randall reasignó el trabajo y ella comenzó a aplicar sus conocimientos sobre fibras de ADN al tiempo que se encargó de dirigir la tesis doctoral de Gosling. El equipo logró, en principio, dilucidar dos formas de ADN, la A y la B. Randall propuso dividir el trabajo y Rosalind eligió enfocarse en la forma A, pero su trabajo la llevó a determinar que ambas formas de ADN estaban conformadas por dos hélices (Sayre, 1975).

Después de dos años de trabajo, Rosalind Franklin dejó el King's College y se integró al Birkbeck College; Wilkins recibió toda la información y las fotografías de la investigación de Rosalind sobre el ADN y, con ella en sus manos, se reunió con James Watson y Francis Crick. El 25 de abril de 1953, Watson y Crick publicaron en la revista Nature un artículo con la descripción de la estructura de doble hélice del ADN (Watson y Crick, 1953), con una nota en la que reconocen el estímulo que tuvieron por el conocimiento de las contribuciones no publicadas de Franklin y Gosling, que están fechadas 16 meses antes (Braun, Tierney y Schmitzer, 2010). En el mismo número de Nature, aparecieron los artículos de Rosalind Franklin y de Wilkins con sus datos de difracción de rayos X, aunque parecen más un apoyo al modelo de la forma B del ADN propuesto por Watson y Crick (Franklin y Gosling, 1953; Wilkins, Strokes y Wilson, 1953). Rosalind Franklin continuó trabajando con la cristalografía de rayos X para determinar, entre otras cosas, la estructura del virus del mosaico del tabaco y de su material genético, es decir, una molécula de ácido ribonucleico (RNA) (Sayre, 1975). 


\section{Mujeres de ciencia en la Actualidad}

A pesar de que muchas cosas han cambiado, la falta en la paridad de género se mantiene -aunque en menor medida- en este siglo pues, a nivel de educación superior, sólo el 35\% de los estudiantes de las áreas de ciencia, tecnología, ingeniería y matemáticas (STEM, por sus siglas en inglés) son mujeres. Desafortunadamente muchas de estas personas abandonan las disciplinas STEM durante sus estudios universitarios, en su transición al mundo laboral e incluso en su trayectoria profesional. Esto ha dado como resultado que actualmente solo el $28 \%$ de todas las personas que hacen investigación científica en el mundo sean mujeres (UNESCO, 2019).

Además de la baja proporción de mujeres en el entorno científico, la supremacía masculina se ha impuesto en el reconocimiento al trabajo realizado por ambos géneros. La presencia femenina comenzó a ser notable en los círculos académicos cuando algunas investigadoras fueron incluidas, aunque de manera minoritaria, en las nominaciones de la Real Academia de Ciencias de Suecia y del Instituto Karolinska a los Premios Nobel. Como se sabe, este es el reconocimiento anual que se concede a personas dedicadas a la ciencia por las investigaciones, los descubrimientos o las contribuciones notables que hacen a la humanidad.

A partir de que se instituyó, el Premio Nobel se otorga en las disciplinas científicas de la Física, la Química y la Fisiología o Medicina. El galardón en cada rama puede ser otorgado a una, dos o tres personas y, hasta ahora, la lista de ganadores está constituida mayoritariamente por hombres, pues solo ha habido 23 mujeres entre los 647 laureados.

Pero ¿cómo ha sido la participación de las mujeres dedicadas a la ciencia respecto al Premio Nobel? Desde que se inició la entrega de este galardón en 1901, hasta el año 2020, el número de veces que lo han recibido hombres en solitario es 38 en el área de la Fisiología, 61 en Química y 47 en Física. El número de ocasiones en que el premio ha sido ganado por grupos formados solo por varones también es elevado, con 62, 45 y 63 veces respectivamente, para las áreas citadas. Mientras que, solamente en 21 ocasiones ha habido mujeres en el podio para recibir el galardón en los campos y en el período de tiempo mencionados; además, sólo en tres de esas ocasiones una mujer lo recibió en solitario (Figura 1) (Nobel Prize, 2021). Es decir que de los 337 premios que se han entregado, únicamente el $0.9 \%$ lo ha recibido una científica sola, sólo el 5.05\% fue otorgado a grupos de investigadores en los que había un integrante del sexo femenino y el $94.05 \%$ restante fue para varones.

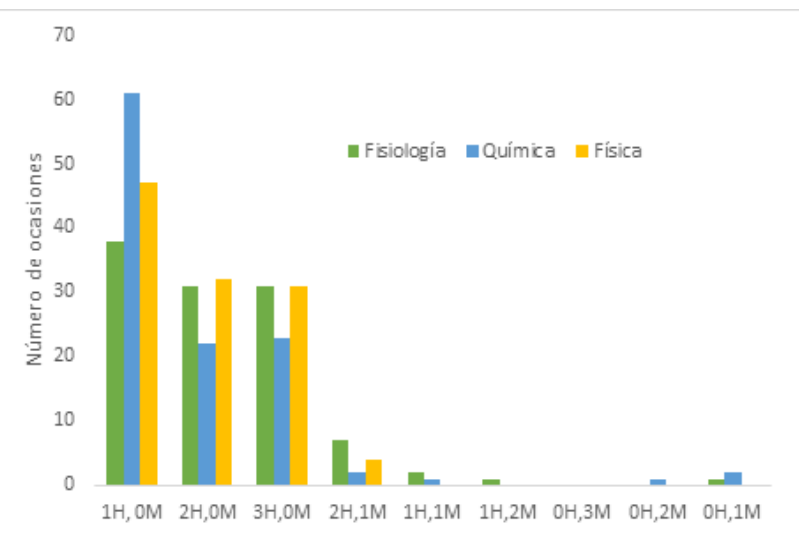

Figura 1. Número de ocasiones de entrega de los Premios Nobel en las áreas de Fisiología o Medicina, Química y Física a: un hombre y cero mujeres (1H,oM); 2 hombres y cero mujeres (2H,oM); 3 hombres y cero mujeres $(3 \mathrm{H}, \mathrm{oM})$; dos hombres y una mujer $(2 \mathrm{H}, 1 \mathrm{M})$; un hombre y una mujer (1H,1M); un hombre y dos mujeres $(\mathbf{1 H}, 2 \mathrm{M})$; cero hombres y tres mujeres $(\mathrm{oH}, 3 \mathrm{M})$; cero hombres y dos mujeres (oH,2M); cero hombres y una mujer (oH,1M).

Además del valor intrínseco que tiene, el galardón está acompañado de un estímulo monetario para que los ganadores puedan continuar sus futuros trabajos sin preocupaciones económicas. Esto implica que un ínfimo número de las mujeres que han ganado el premio han obtenido el monto total de este; en 17 veces las ganadoras compartieron el laurel y la gratificación con varones y solo en una ocasión dos mujeres han dividido el premio entre ellas. Es en este punto donde también se destaca una desigualdad en la entrega del monto económico ya que, cuando se han tenido tres ganadores, el incentivo 
no siempre se ha repartido en tres partes iguales pues algunas veces se ha dividido en dos montos de $25 \%$ y uno de 50\%, y este tipo de repartición ha sido más frecuente en los casos en los que ha habido mujeres galardonadas -que han sido más susceptibles a ganar el 25\%-, mientras que lo más común en la repartición del premio para los hombres ha sido la división en partes iguales (Figura 2).

Figura 2. Porcentaje del estímulo monetario otorgado contra el porcentaje de mujeres y hombres que lo recibieron.

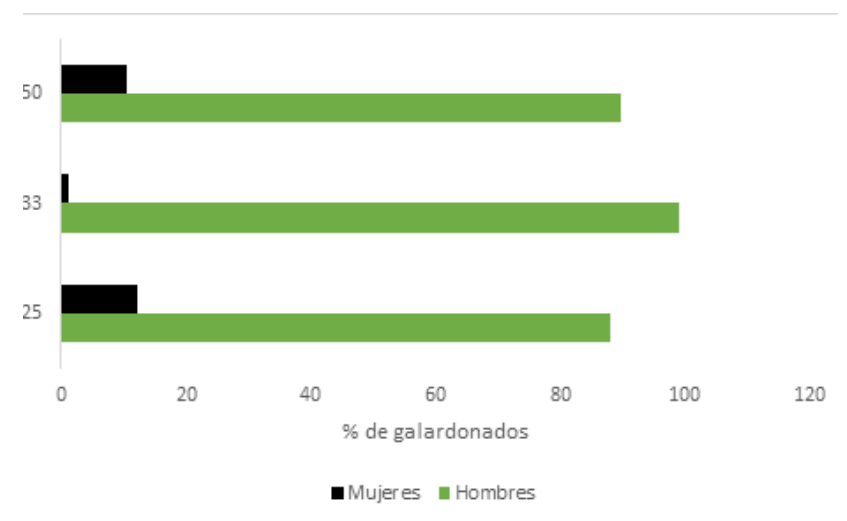

Fuente: Elaboración y análisis propio tomando en cuenta los datos de la página Nobel Prize. (2021).

En una exploración más puntual se destaca que, desde 1901 hasta 2020, la Física es la disciplina con menos ganadoras en Estocolmo, solo cuatro mujeres recibieron el premio y, además, lo compartieron con hombres. En cuanto a la Química se tienen siete galardonadas y en el área de la Fisiología o Medicina, doce.

\section{Ganadoras del Premio Nobel de Física}

En 1903 Marie Curie, su esposo Pierre Curie y Henry Becquerel recibieron el Premio Nobel de Física por su trabajo sobre la radiactividad. En 1895, Becquerel descubrió accidentalmente la radiación natural emitida por el uranio ya que, al colocar sales de este elemento sobre un paquete de placas fotográficas, aisladas de la luz por estar envueltas en papel negro, estas se habían velado. Marie y Pierre Curie investigaron la naturaleza de las radiaciones que producían las sales utilizadas por Becquerel y encontraron que provenían del propio átomo de uranio. Este resultado fue la base para el planteamiento de la teoría de la indivisibilidad de los átomos estudiada en las décadas siguientes y abrió el camino al uso de la radioterapia para destruir tumores cancerígenos (Tomé López, 2015; Merle-Béral, 2018).

6o años después, Maria Geoppert-Mayer compartió el Premio con J. Hans D. Jensen y Eugene Paul Wigner. Maria y Jensen trabajaron en un modelo que describe la estructura interna del núcleo del átomo en donde los protones y neutrones están distribuidos en capas con diferentes niveles de energía. El modelo refleja observaciones de la rotación de los nucleones alrededor de su propio eje, así como también alrededor del centro del núcleo (Nobel Prize, 2020; Merle-Béral, 2018).

Donna Theo Strickland es la tercera mujer en la historia y la primera en el siglo XXI en ganar el Premio Nobel de Física ya que en 2018, ella y Gérard Mourou, recibieron el galardón por el desarrollo de un método de creación de pulsos ópticos de alta resolución, empleados en la actualidad en la realización de cirugías de miopía o cataratas y en la producción de nanopartículas que pueden ser utilizadas para la producción de materiales cuyas propiedades los hacen ideales para la fabricación de microchips, entre otras aplicaciones (Merle-Béral, 2018). Estos dos investigadores compartieron el premio con Arthur Ashkin quien inventó las llamadas pinzas ópticas; estos dispositivos utilizan luz láser para desplazar, atrapar y separar, sin necesidad de contacto físico, partículas del orden de un micrómetro por lo que pueden ser empleados, por ejemplo, en el estudio de las propiedades mecánicas de las membranas celulares in vivo (Ramírez G., 2019).

En el año 2020, se repitió la fórmula de entregar el Premio Nobel de Física a tres personas, dos que trabajaron en conjunto (una mujer y un hombre), y un hombre que trabajó en un proyecto independiente. Andrea Ghez y Reinhard Genzel lo recibieron por el descubrimiento de un objeto compacto supermasivo en el centro de la galaxia. Este hallazgo lo hicieron empleando técnicas de alta resolución espacial en imágenes, como la óptica adaptativa que permite contrarrestar, en tiempo real, los efectos de la atmósfera de la Tierra en la formación de imágenes astronómicas (Nobel Prize, 2021). Con ellos, Roger Penrose recibió el galardón por el descubrimiento 
de que la formación de los agujeros negros es una predicción sólida de la teoría general de la relatividad.

\section{Ganadoras del Premio Nobel de Química}

Aunado a su Premio Nobel de Física recibido en 1903, Marie Curie fue la primera mujer en ganar el Premio Nobel de Química en 1911, obteniéndolo, además, en solitario y es hasta hoy, la única mujer en haber ganado dos de estos galardones en diferentes áreas. La segunda distinción Nobel le fue otorgada por sus servicios para el desarrollo de la química a través del descubrimiento de los elementos radiactivos polonio y radio; además, por el aislamiento del radio y el estudio de la naturaleza y composición de este elemento (Merle-Béral, 2018). Veinticuatro años más tarde, Irène Joliot-Curie, hija de Marie y Pierre Curie, recibió el premio junto con su esposo Frédéric Joliot con quien demostró por primera vez la creación de radioisótopos artificiales por bombardeo de boro, aluminio o magnesio con partículas alfa (núcleos de helio). Este descubrimiento sentó las bases de la reacción en cadena para la construcción de un reactor nuclear que, años después, utilizaría la fisión nuclear controlada para producir energía mediante el uso de uranio y agua pesada (Merle-Béral, 2018; Nobel Prize, 2020). A manera de nota, se debe destacar que la fisión nuclear fue descubierta por la científica austríaca Lise Meitner, quien, sin embargo, nunca fue nominada al Premio Nobel (Vergniory, 2015).

La cristalógrafa Dorothy Crowfoot recibió en solitario el Premio Nobel en 1964 por la determinación mediante rayos $\mathrm{X}$ de la estructura química de sustancias biológicas como la molécula de colesterol, la penicilina, la vitamina B12 y la insulina, entre otras (Merle-Béral, 2018). El conocimiento de la estructura química de estas moléculas fue la base para su síntesis (Téllez Bárdenas, 2006; Martínez Pulido C. , 2016). Cuarenta y cinco años después, los también cristalógrafos Ada Yonath (Merle-Béral, 2018), Venkatraman Ramakrishnan y Thomas A. Steitjuntoz, fueron los ganadores por determinar la estructura de los ribosomas, macromoléculas responsables de la síntesis de las proteínas (Nobel Prize, 2020).

En 2018, Frances Hamilton Arnold compartió el premio con Gregory Winter y George P. Smith, quienes desarrollaron un método para estudiar las interacciones de péptidos expresados de manera exógena en la superficie de virus bacteriófagos. El trabajo de Frances versó sobre la evolución dirigida de las enzimas, proteínas catalizadoras de las reacciones químicas. Los resultados del trabajo de esta investigadora han contribuido en la producción de fármacos y biocombustibles que causan menos daño al medio ambiente (Nobel Prize, 2020).

El trabajo conjunto de dos mujeres fue el motivo para otorgarles el premio en el año 2020. Jennifer A. Doudna y Emmanuelle Charpentier desarrollaron un método para editar secuencias génicas. El método de las tijeras moleculares o CRISPR/Cas9 está basado en el mecanismo de inmunidad adaptativa contra virus y plásmidos que muchas bacterias tienen. $\mathrm{Al}$ ser atacadas bacterias como las arqueas, por ejemplo, son capaces de incorporar en una región de su genoma conocida como CRISPR, fragmentos de material genético provenientes de bacteriófagos o de plásmidos. En un evento posterior la proteasa Cas9, presente en la bacteria, reconoce específicamente los sitios en los que los fragmentos fueron incorporados y los escinde. La simplicidad y especificidad del sistema CRISPR-Casg ha posibilitado el desarrollo de una tecnología precisa y eficiente para, entre otras cosas, editar, modificar, regular y por supuesto estudiar el genoma de los organismos. Sus aplicaciones son muy amplias para la investigación en biología básica, medicina y biotecnología, por ejemplo, en la investigación de nuevos blancos para fármacos, la generación de modelos animales para el estudio de enfermedades e incluso en el desarrollo de estrategias de terapia génica en humanos (Doudna y Charpentier, 2014).

\section{Ganadoras del Premio Nobel de Fisiología o Medicina}

El Premio Nobel de Fisiología o Medicina es el que ha sido otorgado a más mujeres, con un total de 12 galardonadas, aunque sólo Barbara McClintock lo recibió en solitario en 1983, luego de 30 años de haber publicado su trabajo sobre el cambio de posición de genes en los cromosomas del maíz, fenómeno que explica la gran variabilidad en esta planta (Merle-Béral, 2018; Nobel Prize, 2020). 
Gerty Cori y su esposo Carl Ferdinand Cori recibieron el premio en esta área en 1947, compartiéndolo con Bernardo Houssay. Mientras que Houssay lo recibió por sus estudios acerca de la influencia del lóbulo anterior de la hipófisis en la distribución de la glucosa en el cuerpo, el matrimonio Cori recibió el premio por su descubrimiento del mecanismo de la conversión catalítica del glucógeno. El ciclo de Cori explica el proceso por el que el glucógeno, un derivado de la glucosa, se convierte en ácido láctico en el tejido muscular y luego es resintetizado y almacenado como fuente de energía en el hígado; en su momento, el glucógeno es convertido en glucosa y esta es absorbida por las células musculares (Merle-Béral, 2018). Los estudios de estos tres investigadores marcaron un hito en la comprensión de la diabetes (Nobel Prize, 2020).

En 1977, el premio se otorgó a Roger Guillemin, Andrew V. Schally y a Rosalyn Yalow. A los dos primeros se les reconoció por sus descubrimientos sobre la producción de hormonas peptídicas en el cerebro, y a Rosalyn por el desarrollo de una técnica capaz de medir la cantidad de insulina que circula en la sangre (Merle-Béral, 2018). Este método, conocido como radioinmunoensayo, se utiliza para medir cientos de sustancias de interés biológico (Benavente, 2019; Nobel Prize, 2020).

Los descubridores de los factores de crecimiento compartieron el Nobel en 1986. Rita Levi-Montalcini descubrió el Factor de Crecimiento Neuronal y Stanley Cohen el Factor de Crecimiento Epidérmico (NGF y EGF, por sus siglas en inglés, respectivamente). El NGF es un péptido que estimula el crecimiento de las fibras nerviosas y su descubrimiento fue muy importante para el desarrollo de la neurobiología ya que, por primera vez, sustancias de señalización químicamente definidas pudieron ser empleadas en la búsqueda de los mecanismos que regulan el desarrollo neuronal. Por otro lado, el EGF estimula el crecimiento de una gran variedad de células entre las que se incluyen fibroblastos, células hepáticas, vasculares y endocrinas. Su identificación permitió el estudio del proceso normal de crecimiento celular, de su regulación y, por lo tanto, de la transformación celular y el crecimiento de tumores (Deogracias Horrillo, 2016; Merle-Béral, 2018; Nobel Prize, 2020).
Los premios de 1988 y 1995 se otorgaron a dos grupos de tres investigadores cada uno. El primero fue para Gertrude B. Elion, George H. Hitchings y James W. Black, por sus descubrimientos de los principios clave en el tratamiento con medicamentos contra diversas enfermedades, así como por el desarrollo de un método sistemático para la producción de fármacos. Black desarrolló el propanolol y la cimetidina, medicamentos usados para el tratamiento de la hipertensión y para la cura de la úlcera duodenal o de la úlcera de estómago, respectivamente. Mientras que entre las diversas drogas que Gertrude y Hitchings produjeron está la 6-mercaptopurina, un fármaco que inhibe la síntesis de ADN y que pudo ser empleado en el tratamiento contra la leucemia. La colaboración de estos dos científicos se extendió durante 40 años, en los que sintetizaron drogas como el alopurinol, la pirimetamina, y el aciclovir, para el tratamiento de la gota, la malaria y el herpes, respectivamente; además, demostraron la eficacia de la azatiopina como un agente inmunosupresor, mismo que pudo ser usado en los trasplantes de órganos y fue el primero en su tipo (Ruiza, Fernández y Tamaro , 2004; Merle-Béral, 2018; Nobel Prize, 2020).

En el grupo de 1995, Christiane Nüsslein-Volhard compartió el premio con Eric F. Wierschaus y Edward B. Lewis por sus estudios sobre la biología del desarrollo. Con la mosca de la fruta Drosophila melanogaster como modelo de investigación, Christiane y Wierschaus demostraron que la arquitectura de un organismo vivo se construye por etapas y que cada etapa está controlada por un grupo específico de genes. En 1980 identificaron y clasificaron, de entre los miles de genes de la mosca, los 15 que dirigen el desarrollo de un nuevo organismo y le dan la forma tridimensional a su cuerpo (Merle-Béral, 2018; Nobel Prize, 2020). Estos descubrimientos dieron la pauta para que otros investigadores encontraran que muchos de los genes que controlan el desarrollo de la $D$. melanogaster tienen homólogos o equivalentes en otros organismos incluido el ser humano, lo que ha permitido, entre otras cosas, conocer las causas de ciertas anomalías en los embriones humanos (Martínez Pulido C. , 2014).

Linda B. Buck y Richard Axel compartieron el galardón en 2004 luego de que clonaron receptores olfatorios y describieron la organización del sistema del olfato. Su 
trabajo explicó cómo las feromonas y los olores son detectados por los receptores olfatorios localizados en la membrana celular de las neuronas sensoriales del epitelio nasal y cómo son convertidos en impulsos eléctricos que son enviados al cerebro para su interpretación (MerleBéral, 2018; Nobel Prize, 2020).

Cuatro años después, el motivo del otorgamiento del Premio Nobel de Medicina fue el trabajo desarrollado por dos grupos de investigación que durante las décadas de 1970 y 1980 descubrieron a los agentes virales que causan dos terribles enfermedades. En 1976, Harald zur Hausen develó el papel del virus del papiloma humano en el desarrollo del cáncer cérvico-uterino. Por otro lado, en 1983, Francoise Barré-Sinoussi y Luc Montagnier identificaron al virus de la inmunodeficiencia humana que provoca el síndrome de inmunodeficiencia adquirida o SIDA (Merle-Béral, 2018; Nobel Prize, 2020).

El año 2009 fue un buen año para las mujeres en la ciencia, pues el Premio Nobel de Medicina fue otorgado nuevamente a tres personas, siendo dos de ellas mujeres. El trabajo de Elizabeth H. Blackburn, Carol W. Greider y Jack W. Szostak está relacionado con el análisis de la estructura molecular y la organización de los telómeros o extremos terminales de los cromosomas. Los telómeros son regiones repetidas de ADN no codificante del cromosoma encargadas de protegerlo de cualquier daño. Conforme la célula se divide, la secuencia de los telómeros se va acortando hasta el punto en que, debido a su corta longitud, la célula ya no puede dividirse e inicia el camino de la senescencia hasta morir. Aunado a esto, Elizabeth y Carol descubrieron y aislaron a la enzima telomerasa, que es la encargada de producir los telómeros encontrando además que las células cancerosas producen mayor cantidad de telomerasa en comparación con las células normales, lo que ayuda a explicar el hecho de que puedan propagarse indefinidamente (Merle-Béral, 2018; Nobel Prize, 2020).

La historia de un matrimonio ganador del Premio Nobel de Medicina se repitió en 2014 cuando May-Britt Moser y Edvard I. Moser lo ganaron compartiéndolo con John O’Keefe. En 1971 O’Keefe descubrió que, cuando una rata se encontraba en un punto de una habitación ciertas células de su cerebro se activaban y, cuando la rata se movía a otro punto de la estancia, distintas células se accionaban. En 2005, los esposos Moser encontraron en el hipocampo -un área localizada en el centro del cerebro-, un tipo de células nerviosas que coordinan un sistema de navegación que permite a un organismo ubicarse en el espacio. También demostraron cómo este tipo de células cooperan entre ellas para lograr su objetivo (Merle-Béral, 2018; Nobel Prize, 2020).

Por último, la científica china Tu Youyou compartió el premio con Satoshi Omura y William Campbel en 2015. Los trabajos de Omura y Campbel se refieren al descubrimiento de terapias nuevas para el combate de infecciones como la oncocercosis y la filariasis linfática causadas por parásitos. Por otro lado, Tu Youyou encaminó sus estudios hacia la investigación de la medicina tradicional china y en 1970, extrajo de la planta Artemisia annua la sustancia artemisina, capaz de inhibir el desarrollo del parásito que causa la malaria, una de las enfermedades más letales que la humanidad ha conocido. El desarrollo de las drogas basado en la artemisina ha sido determinante en la sobrevivencia y mejoramiento de las condiciones de salud de las personas afectadas por malaria (Merle-Béral, 2018; Nobel Prize, 2020).

\section{Otras mujeres ganadoras del Premio Nobel}

En esta exploración sobre el reconocimiento al trabajo de mujeres científicas usando como referencia el otorgamiento del Premio Nobel, hasta el año 2020, el escaso número de investigadoras reconocidas con el galardón exhibe las menguas cifras de mujeres participantes en las áreas científicas y el poco reconocimiento a su trabajo. Una de las razones de esta situación es que en el pasado se consideraba -de manera lamentable- que las mujeres no eran académicamente competentes y que de ninguna manera estaban o podían estar al nivel de los hombres. Esto cerró las puertas a niñas y jóvenes para acceder a una preparación académica superior. A pesar de que ese horizonte ha cambiado, actualmente sólo el $28 \%$ de las personas que se dedican a la ciencia en el mundo son mujeres; aun así, las investigaciones realizadas por ellas han contribuido al enriquecimiento de la ciencia. 
Finalmente, echando un vistazo a las tres áreas restantes en las que se otorga el Premio Nobel vemos que el panorama no cambia mucho, pues a lo largo de más de 100 años, el galardón de Literatura se ha otorgado sólo en 16 ocasiones a mujeres. El premio Nobel de la Paz lo han ganado 17 mujeres en 14 ocasiones, seis de ellas en solitario, cinco lo compartieron con un varón y una con una coalición internacional, mientras que en dos ocasiones ha habido más de una ganadora, dos en 1976 y tres en 2011. En el área de la Economía, el galardón ha sido entregado desde hace 50 años y, en el podio solo ha habido dos mujeres que además lo compartieron con varones. Esto da un total de 57 ganadoras hasta la premiación de 2020 (Nobel Prize, 2021). Sin embargo, se ha de destacar que, como se mencionó antes, 2009 fue un año especialmente bueno para las mujeres en Suecia pues ha sido en el que más galardonadas ha habido: Ada Yonath, Elizabeth H. Blackburn y Carol W. Greider en las disciplinas científicas, y Elinor Ostrom y Herta Müller en las áreas de Economía y Literatura, respectivamente, aunque solo la última recibió el galardón en solitario.

\section{Conclusión}

Desde tiempos muy antiguos, los hombres han tenido control sobre las mujeres por la implementación de un dominio físico, sexual, social e intelectual. Esta dominación ha sido la causa de que el desarrollo académico de eminentes mujeres haya sido bloqueado. El grado de analfabetismo de las mujeres en el pasado las orillaba a ser dependientes, muchas veces de manera total, de los hombres. Por fortuna, hubo mujeres que tuvieron la oportunidad de acceder a una educación académica formal y con ello demostraron que no hay diferencias intelectuales entre hombres y mujeres (Frize, 2013). Y, aunque también se tiene registro de hombres que apoyaron a sus esposas e hijas en su desarrollo personal, académico, intelectual y profesional (Frize, 2013; Duarte Cruz y García-Horta, 2016), lamentablemente el curso de los siglos no ha cambiado la mentalidad de hombres misóginos que no reconocen las capacidades femeninas (García Dauder y Pérez Sedeño, 2017).

En cuanto a la ciencia es innegable que, en el nuevo milenio el acceso de las mujeres a la educación superior y su presencia en los laboratorios de investigación científica se ha incrementado, aunque todavía se tiene un porcentaje muy bajo de investigadoras en el mundo. Además, el trabajo de las mujeres ha sido opacado durante décadas por notables varones a pesar de que sus colaboraciones a la ciencia han sido igual de valiosas que las de ellos o incluso más. Un ejemplo claro de esto es el caso de Rosalind Franklin, quien realizó los estudios que dilucidaron la estructura molecular del ADN, la molécula que guarda y transfiere la información genética de los organismos y que, sin embargo, no fue considerada para la entrega del galardón que, en 1962, fue otorgado a Watson, Crick y Wilkins.

El bajo número de mujeres que han sido reconocidas con el Premio Nobel puede deberse, como ya se dijo, a la baja participación femenina en los campos de la ciencia, la ingeniería y las matemáticas, sin embargo, en el inicio de la tercera década del siglo XXI y con los años por venir en los que, indudablemente, habrá muchas preguntas científicas que responder o tecnologías que desarrollar, además de la creciente participación femenina en la academia, es altamente probable que se vean, dichosamente, más ganadoras en Suecia.

\section{Referencia bibliográfica}

Benavente, R. (19 de Septiembre de 2019). Rosalyn Yalow, la biofísica testaruda que descubrió cómo medir la insulina en la sangre (y ganó un Nobel por ello). Recuperado el 2 de Abril de 2020 de https:// www.mujeresconciencia.com/2019/o9/19/ rosalyn-yalow-la-biofísica-testaruda-quedescubrio-como-medir-la-insulina-en-la-sangrey-gano-un-nobel-por-ello/

Braun, G., Tierney, D., y Schmitzer, H. (2010). How Rosalind Franklin Discovered the Helical Structure of DNA: Experiments in Diffraction. The Physics Teacher. https://doi:10.1119/1.3555496

Camarena Adame, M., y Saavedra García, M. (2018). El techo de cristal en México. La Ventana, (47), 312347 . 
de Bingen, H. Libro de las obras divinas. Titivillus.

Deogracias Horrillo, M. (19 de Diciembre de 2016). La imperfección como motivación vital. Recuperado el 2 de Abril de 2020 de https:// www.mujeresconciencia.com/2016/12/19/laimperfeccion-motivacion-vital/

Doudna, J., y Charpentier, E. (2014). The new frontier of genome engineering with CRISPR-Cas9. Science, 346(6213), 1077. https://doi:10.1126/ science. 1258096

du Châtelet. (1996). Discurso sobre la felicidad y Correspondencia. Cátedra.

Duarte Cruz, J., y García-Horta, J. (2016). Igualdad, Equidad de Género y Feminismo, una mirada histórica a la conquista de los derechos de las mujeres. Revista CS, (18), 107-158.

Dzielska, M. (2004). Hipatia de Alejandría. Siruela.

Essinger, J. (2014). El algoritmo de Ada. La vida de Ada Lovelace, hija de lord Byron y pionera de la era informática. Un_Tal_Lucas.

Franklin, R., y Gosling, R. (1953). Molecular Configuration in Sodium Thymonucleate. Nature, 171(4356), 740-741. https://doi:10.1038/171740ao

Frize, M. (2013). Laura Bassi and Science in 18th Century Europe. The extraordinary Life and Role of Italy's Pioneering Female Professor. Springer.

García Dauder, S., y Pérez Sedeño, E. (2017). Las "mentiras" científicas sobre las mujeres. Los Libros de la Catarata.

Gregersen, E. (16 de Frebrero de 2011). Laura Bassi. https://doi:doi:10.1086/356547

Griffith, F. (1928). The significance of Pneumococcal types. Journal of Hygiene, 27(2), 113-59.
Martínez Pulido, C. (9 de Julio de 2014). ¿Cómo se desarrolla un embrión? Responde Christiane Nüsslein-Volhard, Premio Nobel alemana. Recuperado el 6 de Abril de 2020 de https:// www.mujeresconciencia.com/2014/07/o9/comose-desarrolla-un-embrion-responde-christianenusslein-volhard-premio-nobel-alema/

Martínez Pulido, C. (11 de mayo de 2016). Capturada por la química: Dorothy Crowfoot Hodgkin. Recuperado el 1 de Abril de 2020 de https://mujeresconciencia. com/2016/05/11/capturada-la-quimica-dorothycrowfoot-hodgkin/

Merle-Béral, H. (2018). 17 mujeres Premios Nobel de ciencias. Plataforma Editorial.

Nobel Prize. (2020). Ada E. Yonath - Facts. Recuperado el 2 de abril de 2020 de https://www.nobelprize. org/prizes/chemistry/2009/yonath/facts/

Nobel Prize. (2020). Barbara McClintock-Facts. Recuperado el 2 de abril de 2020 de https://www. nobelprize.org/prizes/medicine/1983/mcclintock/ facts/

Nobel Prize. (2020). Carol W. Greider - Facts. Recuperado el 11 de abril de 2020 de www.nobelprize.org/ prizes/medicine/2009/greider/facts/

Nobel Prize. (2020). Christiane Nüsslein-Volhard - Facts. Recuperado el 6 de abril de 2020 de https://www. nobelprize.org/prizes/medicine/1995/nussleinvolhard/facts/

Nobel Prize. (2020). Frances H. Arnold - Facts. Recuperado el 2 de abril de 2020 de https://www.nobelprize. org/prizes/chemistry/2018/arnold/facts/

Nobel Prize. (2020). Francoise Barré-Sinoussi - Facts. Recuperado el 11 de abril de 2020 de https:// www.nobelprize.org/prizes/medicine/2008/ barre-sinoussi/facts/ 
Nobel Prize. (2020). Gertrude B. Elion - Facts. Recuperado el 5 de abril de 2020 de https://www.nobelprize. org/prizes/medicine/1988/elion/facts/

Nobel Prize. (2020). Gerty Cori - Facts. Recuperado el 2 de abril de 2020 de https://www.nobelprize.org/ prizes/medicine/1947/cori-gt/facts/

Nobel Prize. (2020). Irène Joliot-Curie-Facts. Recuperado el 1 de abril de 2020 de https://www.nobelprize. org/prizes/chemistry/1935/joliot-curie/facts/

Nobel Prize. (2020). Linda B. Buck - Facts. Recuperado el 6 de abril de 2020 de https://www.nobelprize. org/prizes/medicine/2004/buck/facts/

Nobel Prize. (2020). Maria Goeppert Mayer - Facts. Recuperado el 2 de abril de 2020 de https://www. nobelprize.org/prizes/physics/1963/mayer/facts/

Nobel Prize. (2020). May-Britt Moser - Facts. Recuperado el 11 de abril de 2020 de https://www.nobelprize. org/prizes/medicine/2014/may-britt-moser/ facts/

Nobel Prize. (2020). Rita Levi-Montalcini - Facts. Recuperado el 2 de mayo de 2020 de https:// www.nobelprize.org/prizes/medicine/1986/levimontalcini/facts/

Nobel Prize. (2020). Rosalyn Yalow - Facts. Recuperado el 2 de abril de 2020 de https://www.nobelprize. org/prizes/medicine/1977/yalow/facts/

Nobel Prize. (2020). The Nobel Prize in Physiology or Medicine 2009. Recuperado el 11 de abril de 2020 de https://www.nobelprize.org/prizes/ medicine/2009/summary/

Nobel Prize. (2020). Tu Youyou - Facts. Recuperado el 11 de abril de 2020 de https://www.nobelprize.org/ prizes/medicine/2015/tu/facts/
Nobel Prize. (2021). Andrea Ghez - Facts. Recuperado el 19 de abril de 2021 de https://www.nobelprize. org/prizes/physics/2020/ghez/facts/

Nobel Prize. (2021). The Nobel Prize. Recuperado el 19 de abril de 2021 de https://www.nobelprize.org/

Ramírez G., C. (2019). Pinzas ópticas y pulsos ópticos ultracortos: un Nobel bien merecido. Momento, (58), 1-7.

Ruiza, M., Fernández, T., y Tamaro , E. (2004). Biografía de Gertrude Belle Elion. Recuperado el 5 de Abril de 2020, de Biografías y Vidas. La enciclopedia biográfica en linea.: https://www. biografiasyvidas.com/biografia/e/elion.htm

Sayre, A. (1975). Rosalind Franklin and DNA. Norton.

Téllez Bárdenas, F. (2006). Mujeres en la cristalografía. Clepsydra, (5), 103-110.

Tomé López, C. (13 de Enero de 2015). El arduo camino al Nobel de Marie Curie. Recuperado el 30 de Marzo de 2020, de Químicos modernos Experientia docet: https://mujeresconciencia. com/2015/og/14/el-arduo-camino-al-nobel-demarie-curie/

UNESCO. (2019). Descifrar el código: La educación de las niñas y las mujeres en ciencias, tecnología, ingeniería y matemáticas (STEM). París, Francia. Obtenido de https://unesdoc.unesco.org/ ark:/48223/pfoooo366649

Vergniory, M. G. (2015). Lise Meitner, la científica que descubrió la fisión nuclear. Recuperado el 21 de mayo de 2020 de https://mujeresconciencia. com/2015/03/o4/lise-meitner-la-cientifica-quedescubrio-la-fision-nuclear/

Watson, J., y Crick, F. (1953). Molecular structure of nucleic acids. Nature, 171(4356), 737-738. https:// doi:10.1038/171737ao 
Wilkins, M., Strokes, A., y Wilson, H. (1953). Molecular structure of deoxypentose nucleic acids. Nature, 171(4356), 738-740. https://doi:10.1038/171738ao 